\title{
Microgrids as Flexible and Network-Connected Grid Assets in Active Distribution Systems
}

\author{
Lei Shao, Xu Zhou, Ji Li (D), Hongli Liu, and Xiaoqi Chen \\ Tianjin Key Laboratory for Control Theory \& Application in Complicated Systems, Tianjin University of Technology, \\ Tianjin 300384, China \\ Correspondence should be addressed to Ji Li; liji0606@163.com
}

Received 3 May 2018; Revised 12 June 2018; Accepted 19 June 2018; Published 24 July 2018

Academic Editor: Mustafa I. Fadhel

Copyright (C) 2018 Lei Shao et al. This is an open access article distributed under the Creative Commons Attribution License, which permits unrestricted use, distribution, and reproduction in any medium, provided the original work is properly cited.

The functionalities of microgrids are detailed and thereby expanded in active distribution systems. A versatile and coordinated operation among multiple microgrids is proposed to facilitate the integration of renewable energy sources (RES) in modern distribution grids. Particularly, to meet the requirements of high penetration level of renewables (e.g., photovoltaics and small hydro), more than one networked controlled microgrids are deployed simultaneously in different locations of a distribution system. Therefore, local RES can be aggregated in each microgrids. In order to satisfy the latest standards in terms of renewable energy integration (i.e., IEEE Std. $1547 \mathrm{Rev}$ ), an optimal operation strategy is designed to coordinate the operation of multiple microgrids. A simulation model is implemented in MATLAB to validate the proposed networked microgrids and the corresponding operation strategy.

\section{Introduction}

The existing planning and operation issues with respect to traditional fossil fuels have drawn increasing attention nowadays. These include the inevitable environmental pollution, increasing $\mathrm{CO}_{2}$ emission, ever-decreasing fuel, and energy. In order to solve the present energy crisis, a paradigm shift in energy configuration should be initialized. Therefore, renewable energy sources (RES) have been gradually involved in the portfolio of energy deployment [1-3]. Compared to conventional energy sources, RES play a significant role in releasing the environmental stress in the whole energy cycle, including generation, transmission, distribution, and consumption. For example, large-scale wind farm can be used to combine with legacy thermal generation units in the power generation system, while high penetration level of different types of renewables is being used in the transmission and distribution grids. At the customer's side, residential renewables are also widely used to localize energy generation and consumption so that an active 'prosumer' is implemented.

Even though the increasing penetration level of RES has effectively solved the environmental issues in the past years and their advantages are obviously seen in modern grid operation, there are still issues that need further attention. Most importantly, it should be noticed that the RES does not have sufficient inertia, which is quite different from conventional synchronous generator based power systems and highly challenges the system stability [4-6]. To solve this potential stability issues, many approaches have been proposed and deployed in both academia and field applications. Energy storage systems (ESS) have been widely employed to compensate the intermittency in output power, which is induced by the low-inertia RES [7]. Hybrid energy sources, such as combined electrical and thermal generation systems, are being studied and gradually used in the actual power systems to leverage their complementary dynamic characteristics and mitigate the variations in their aggregated output power [8]. Meanwhile, it is worth mentioning that, to effectively integrate different types of sources and loads in a localized way, microgrids are being intensively studied over the past years [9-11]. A microgrid can be regarded as an integrated unit with both distributed sources and loads in it, and, in the meantime, with a point of common coupling (PCC), it can be regarded as a controllable unit and the output voltage, current, or power at the PCC can be controlled and flexibly adjusted. Given the tremendous advantages of 
microgrids compared to dispersed RES, microgrids have been used in many field test sites, including mission-critical applications (e.g., isolated power systems in airplanes), remote areas, or, most frequently, grid-connected systems with high requirements of power quality.

Note that the existing penetration level of RES in modern electric grids is high and the percentage of renewable energy in the total energy portfolio keeps increasing due to the evergrowing needs in electricity. Therefore, the current study of microgrids has been greatly advanced at the present. In the first stage, the research on microgrids mainly stays in individual one. In other words, single microgrids are the major research focus. However, with large-scale integration of RES, single microgrid integration cannot always meet the requirements. Hence, people are considering to further advance the concept of microgrids and proposing new technologies and expand the research and application horizon of microgrid deployment. Among the technologies, networked microgrid stays as one of the promising candidates [12]. Different from conventional single microgrids, networked microgrids enable multiple microgrids to coexist in the electric grids and coordinate with each other following a predesigned operation condition. Particularly, being geographically diversified, these networked microgrids can be distributed into different areas of a whole electric grids, so that the integration of renewables can be implemented in a simpler way. Effective control and coordination among multiple microgrids should be well established. Therefore, the penetration level of renewables in various areas of an electric grid can be balanced.

In this paper, the latest operation requirements of electric grids with high penetration level of renewable energy are reviewed along with the versatile functionalities of microgrids. Meanwhile, a problem formulation is established focusing on networked microgrids, especially for the coordinated operation among multiple microgrids. The interactions among multiple microgrids controllers are studied and modeled in the above problem formulation. A simulation test based on MATLAB is built up to validate the proposed coordinated control strategy.

The remainder of the paper is summarized below. Section 2 formulates the problem and defines the networked microgrids under study. Section 3 establishes the optimization formulation of networked microgrids to achieve the optimal operation in active distribution grids. The detailed constraints and optimal objectives are listed to implement the comprehensive formulation. Simulation models of 28-bus and 123-bus test systems rebuilt up in MATLAB in Section 4 to verify the proposed algorithm. Finally, Section 5 draws the conclusion of the whole paper.

\section{Problem Formulation and Definition of Networked Microgrids}

As in the precedent discussion, microgrids can be used as versatile units in distribution systems to facilitate the integration of RES and enhance the performance of distribution operation. Conventional RES is commonly integrated into distribution grids in a dispersed way, which is hard to control
TABLE 1: Variables and parameters.

\begin{tabular}{|c|c|}
\hline$p_{\mathrm{dp}, \mathrm{i}}$ & Dispatchable power \#i \\
\hline$q_{\mathrm{dp}, \mathrm{i}}$ & Dispatchable reactive power $\# \mathrm{i}$ \\
\hline$p_{1, \mathrm{ex}}$ & Injected power into the microgrid \\
\hline$p_{2, \mathrm{ex}}$ & Output power from the microgrid \\
\hline$p_{d, \mathrm{i}}$ & Load active power $\# \mathrm{i}$ \\
\hline$q_{d, \mathrm{i}}$ & Load reactive power $\# \mathrm{i}$ \\
\hline$p_{\text {ndp,i }}$ & PV or other non-dispatchable output power \#i \\
\hline$c_{g}$ & Cost: dispatchable load \\
\hline$c_{e}$ & Cost: power exchange \\
\hline$c_{d}$ & Cost: demand side \\
\hline$V_{\mathrm{i}}$ & Voltage \#i \\
\hline$V_{\varepsilon}$ & Maximum voltage deviation \\
\hline$P_{\mathrm{i}}$ & Active power flow \#i \\
\hline$Q_{\mathrm{i}}$ & Reactive power flow \#i \\
\hline$p_{\mathrm{i}}^{\max }$ & Maximum output active power \\
\hline$q_{\mathrm{i}}^{\max }$ & Maximum output reactive power \\
\hline$r_{\mathrm{i}}$ & Line resistance \\
\hline$x_{\mathrm{i}}$ & Line inductance \\
\hline
\end{tabular}

and manage and may induce unexpected operational issues. To better utilize their advantages, networked microgrids can be deployed to achieve the optimal and coordinated control, as depicted in Figure 1. The problem formulation of networked microgrids is established based on using multiple interconnected microgrids to achieve aggregation of local DERs. Therefore, these DERs can be better operated and controlled without violating any operation constraints, e.g., potential voltage violations and active and reactive power violations. For a clear narrative, the related system parameters and variables are shown in Table 1.

\section{Model Development of Networked Microgrids}

The objective of the optimization problem is established to minimize the cost for exchanging power locally and between the network-connected microgrids. As shown below in (1), the first two terms represent the cost of local sources and loads, and the third to sixth terms show the cost induced by power exchange between networked microgrids. Note that the last two terms in (1) are only used when solving the optimization algorithm, which can be regarded as penalty items to enhance the convergence rate of the optimization problem.

$$
\begin{aligned}
\min & \sum_{i \in N} c_{g} p_{d p, i}-\sum_{i \in N} c_{d} p_{d, i} \\
& +\left(c_{e} p_{1, e x}+\sum_{m} c_{e} p_{1, e x}^{m}-c_{e} p_{2, e x}-\sum_{m} c_{e} p_{2, e x}^{m}\right)
\end{aligned}
$$

The power balance is the most critical constraint that needs to be considered in the optimization problem. As shown in (2), given the flexibility of the output power of the sources and the power consumption of the loads, the 


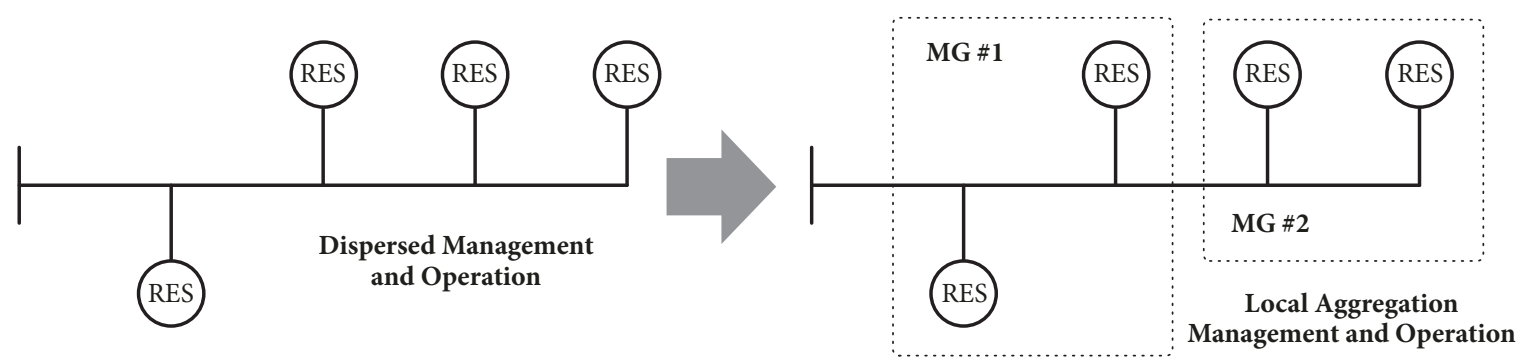

FIGURE 1: Conventional RES integration and the configuration with networked microgrids.

total power generation should be no less than the total load consumption. Note that the power sources include the local power generation units (e.g., the dispatchable sources) and the power injection from the other microgrids.

$$
\begin{gathered}
\sum_{i \in N} p_{d p, i}+\sum_{i \in N} p_{n d p, i}+\sum_{m} p_{1, e x}^{m}+p_{1, e x} \\
\geq \sum_{i \in N} p_{d, i}+\sum_{m} p_{2, e x}^{m}+p_{2, e x}
\end{gathered}
$$

The distribution model should be established in the formulation so that the variations and limitations induced by the model itself can be considered. These constraints are formulated as shown in (3)-(5). Note that, in order to maintain a convex optimization problem, the distribution model is approximated and linearized using the format shown in (3)-(5), which follows the typical linearization in DistFlow.

$$
\begin{aligned}
P_{i+1} & =P_{i}-p_{d, i+1}+p_{n d p, i+1}+p_{d p, i+1} \\
Q_{i+1} & =Q_{i}-q_{d, i+1}+q_{d p, i+1} \\
V_{i+1} & =V_{i}-\frac{\left(r_{i} P_{i}+x_{i} Q_{i}\right)}{V_{1}}
\end{aligned}
$$

Besides the constraints above, it is also necessary to consider the operation limitations of each source, i.e., the voltage and power generation limits, as detailed in (6)-(8). Note that to maximize the power generation of nondispatchable sources, such as solar generation units and wind turbines, the output power of nondispatchable units is not limited. In other words, the output power of the nondispatchable unit follows its maximum power point and is not controlled.

$$
\begin{aligned}
1-V_{\varepsilon} & \leq V_{i} \leq 1+V_{\varepsilon} \\
0 & \leq p_{d p, i} \leq p_{i}^{\max } \\
0 & \leq q_{d p, i} \leq q_{i}^{\max }
\end{aligned}
$$

In the meantime, a penalty term is involved to facilitate the convergence of the formulated optimization problem. To limit these penalty terms, the power exchange determined in each microgrid controller is monitored and their differences are controlled within the acceptable limits.

Therefore, by considering the above formula together, the optimization formulation can be established as below and the corresponding constraints and objectives can be summarized in Figure 2.

$$
\begin{array}{ll}
\min \quad & \sum_{i \in N} c_{g} p_{d p, i}-\sum_{i \in N} c_{d} p_{d, i} \\
& +\left(c_{e} p_{1, e x}+\sum_{m} c_{e} p_{1, e x}^{m}-c_{e} p_{2, e x}-\sum_{m} c_{e} p_{2, e x}^{m}\right) \\
\text { s.t. } \quad & \sum_{i \in N} p_{d p, i}+\sum_{i \in N} p_{n d p, i}+\sum_{m} p_{1, e x}^{m}+p_{1, e x} \\
& \geq \sum_{i \in N} p_{d, i}+\sum_{m} p_{2, e x}^{m}+p_{2, e x} \\
& P_{i+1}=P_{i}-p_{d, i+1}+p_{n d p, i+1}+p_{d p, i+1} \\
& Q_{i+1}=Q_{i}-q_{d, i+1}+q_{d p, i+1} \\
& V_{i+1}=V_{i}-\frac{\left(r_{i} P_{i}+x_{i} Q_{i}\right)}{V_{1}} \\
& 1-V_{\varepsilon} \leq V_{i} \leq 1+V_{\varepsilon} \\
0 \leq p_{d p, i} \leq p_{i}^{\max } \\
0 \leq q_{d p, i} \leq q_{i}^{\max }
\end{array}
$$

\section{Simulation Verification and Validation}

4.1. Small-Scale Test System: 28-Bus Test Feeder. In order to verify the proposed optimization formulation and minimize the operation cost using networked microgrids, a numerical model is established in MATLAB. The configuration of the testing system is shown in Figure 3.

As depicted in Figure 3, the testing feeder is a 28 bus system. In addition to the conventional passive loads and power cables between neighboring buses, six DGs are incorporated in the system to further study their impacts on the system and the effectiveness of using MGs to aggregate the local sources and loads. Particularly, there are two MGs in the system. MG \#1 locates near the feeder head, while MG \#2 locates near the feeder end. Besides these two MGs, two additional DGs are also dispersedly connected to the main feeder. The system parameter is shown in Table 2.

By running the overall comprehensive optimization problem in (9), the results can be summarized in Table 3, where the output power of each dispatchable sources are calculated. 


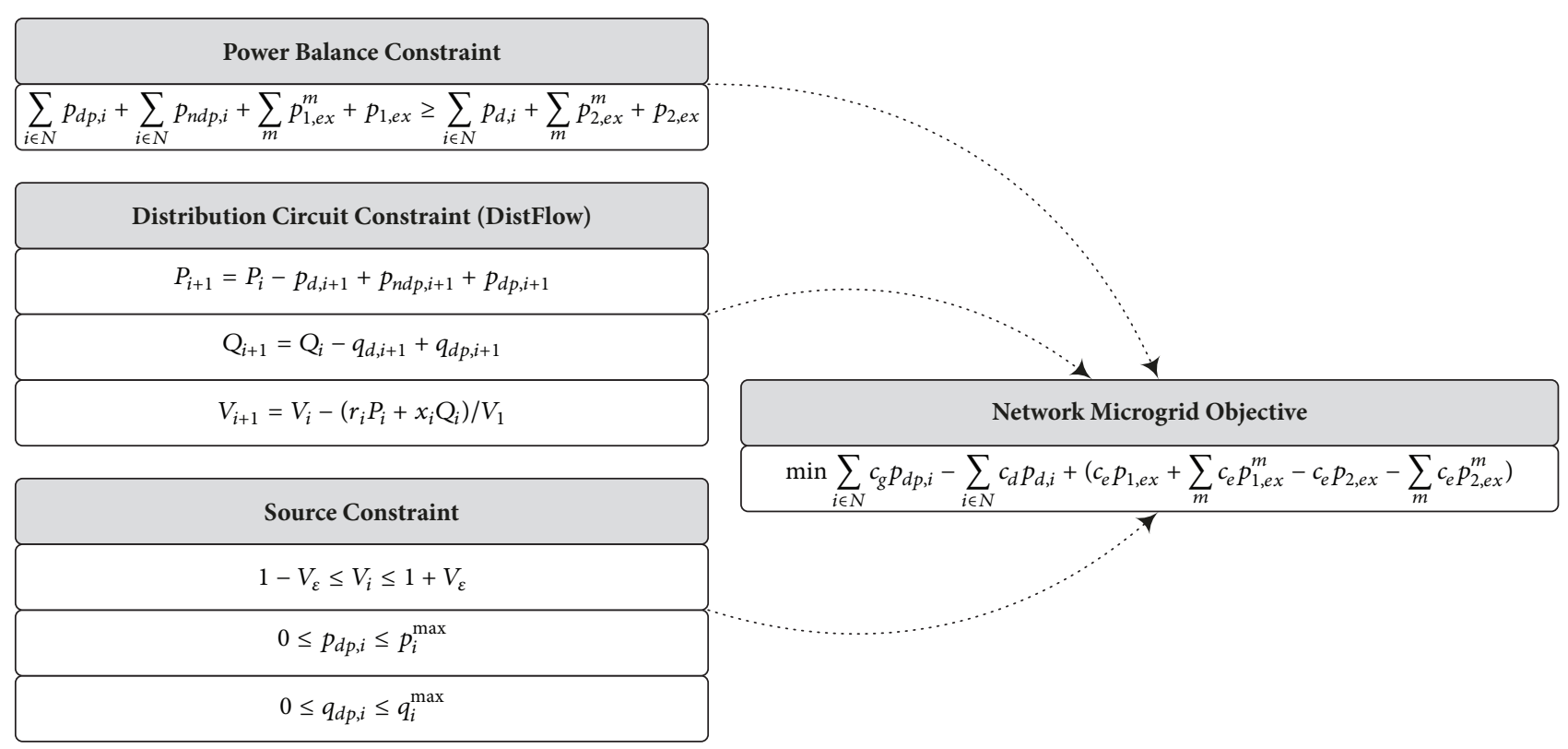

FIGURE 2: Optimization model of networked microgrids.

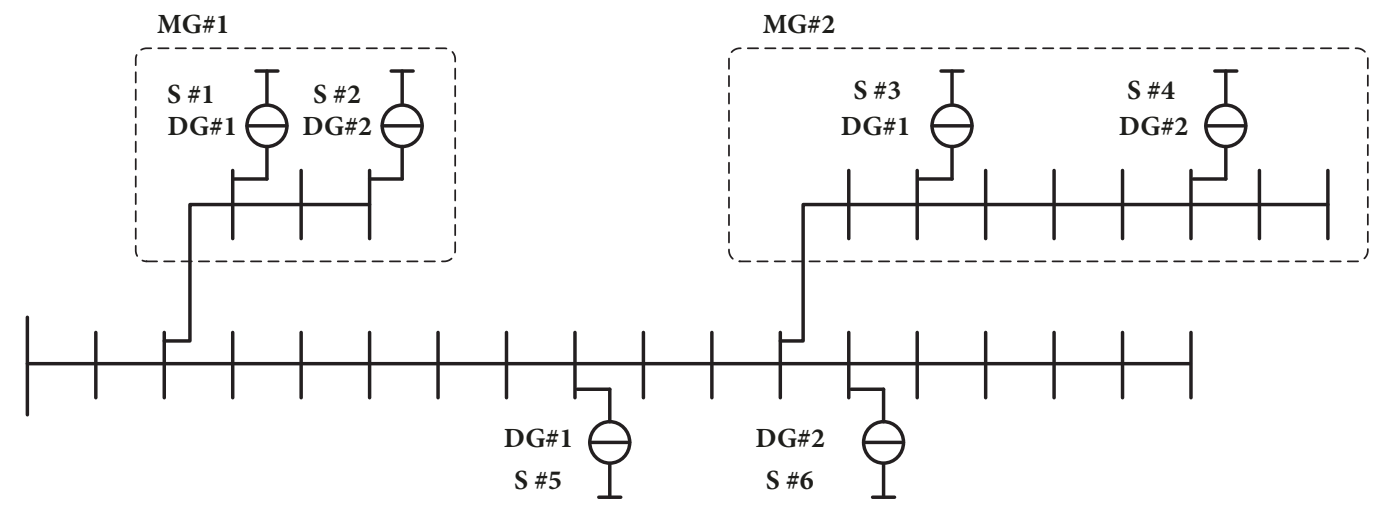

Figure 3: Configuration of the testing system.

TABLE 2: System parameters.

\begin{tabular}{lcc}
\hline Parameter & Value & Unit \\
\hline Base power & 1 & $\mathrm{MW}$ \\
Base voltage & 110 & $\mathrm{kV}$ \\
Line resistance & 0.014 & p.u. \\
Line inductance & 0.026 & p.u. \\
Active load in MG \#1 & 2 & p.u. \\
Active load in MG \#2 & 1.8 & p.u. \\
Active load outside the two MGs & 2 & p.u. \\
Cost: dispatchable load & 0.12 & $\$ / \mathrm{kW}$ \\
Cost: power exchange & 0.2 & $\$ / \mathrm{kW}$ \\
Cost: demand side & 0.4 & $\$ / \mathrm{kW}$ \\
\hline
\end{tabular}

Here, sources \#1 and \#2 belong to microgrid \#1, while sources $\# 3$ and \#4 belong to microgrid \#2. It is seen that the output power converge in the first three steps.
Meanwhile, maximum and minimum of the output power and voltage amplitude in each microgrid are depicted in Figures 4(a) and 4(b). Per unit values are used here. It can be seen that the output power and voltage do not violate their upper and lower boundaries during the interaction of the optimization algorithm.

It should be noticed that, by using the proposed approach, the bus voltage throughout the test system can be well regulated within the acceptable range, and the output power of DGs can finally converge without triggering any stability issues. Conventional approaches still lack of consideration of microgrids and DGs when regulating voltage and power in distribution grids, which will lead to voltage violation and the sequential misfunctional behavior of protective devices. By using the proposed method, the DGs and microgrids can be well controlled and managed to contribute to voltage regulation in distribution grids. As a further numerical study, the proposed system configuration and the conventional system without microgrids (i.e., with all the microgrids 
TABLE 3: Power generation of each source (p.u.).

\begin{tabular}{|c|c|c|c|c|c|}
\hline Interaction & 1 & 2 & 3 & 4 & 5 \\
\hline Source \#1 (MG \#1) & 0.837 & 0.801 & 0.792 & 0.792 & 0.792 \\
\hline Source \#2 (MG \#1) & 0.96 & 0.936 & 0.882 & 0.882 & 0.882 \\
\hline Source \#3 (MG \#2) & 0.882 & 0.864 & 0.774 & 0.774 & 0.774 \\
\hline Source \#4 (MG \#2) & 0.948 & 0.858 & 0.846 & 0.846 & 0.846 \\
\hline Source \#5 & 0.891 & 0.864 & 0.846 & 0.846 & 0.846 \\
\hline Source \#6 & 0.846 & 0.81 & 0.792 & 0.792 & 0.792 \\
\hline
\end{tabular}

TABLE 4: Voltage profile comparison between conventional approach with only dispersed DGs and the developed approach with networked microgrids (p.u.).

\begin{tabular}{lcccc}
\hline & Source \#1 & Source \#2 & Source \#3 & Source \#4 \\
\hline w/ Networked Microgrids & $\mathbf{1 . 0 2 0 0}$ & $\mathbf{1 . 0 2 2 0}$ & $\mathbf{0 . 9 8 2 0}$ & 0.9800 \\
w/o Networked Microgrids & 0.9989 & 1.0023 & 0.9960 \\
\hline
\end{tabular}

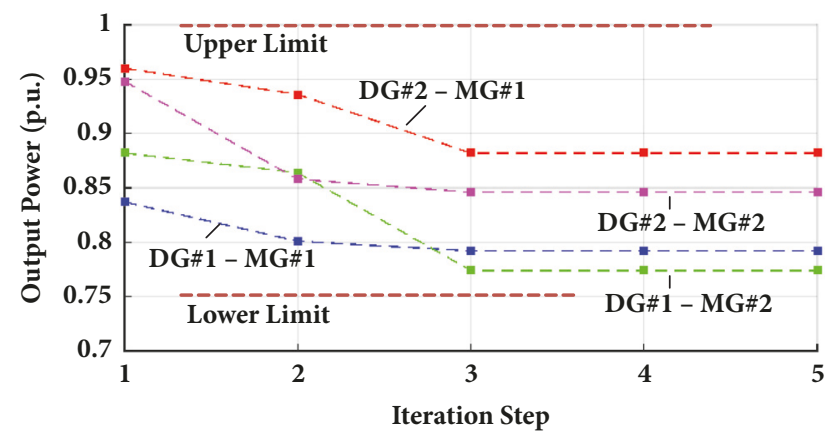

(a)

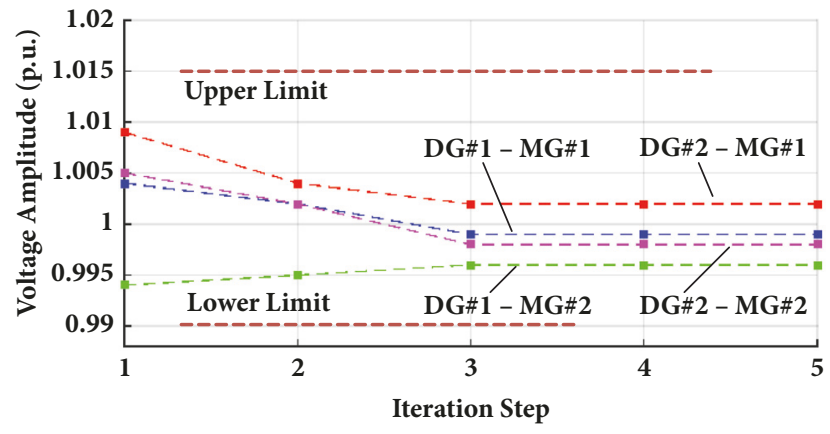

(b)

FIGURE 4: Maximum and minimum of power and voltage during interaction. (a) Output power. (b) Voltage amplitude.

in Figure 3 removed and only keeping the DGs in the system), the results of the comparative study are shown in Table 4. It can be seen that the final voltage values violate the upper or lower boundary at DG \#1 and \#2 since the dispatchable sources cannot be successfully managed, and more importantly, they cannot be coordinated using networked microgrids to maintain the acceptable voltage level. Therefore, the power injected from some DGs violates the upper voltage limit (1.015 p.u.) by injecting higher power into the point of connection, while some other DGs violate the lower voltage limit $(0.9900$ p.u. $)$ by injecting insufficient power into the point of connection. The verification and validation of the proposed method are further demonstrated in the above numerical results.

4.2. Large-Scale Test System: 123-Bus Test Feeder. After the testing in the small-scale 28-bus system, a test in largerscale test feeder, i.e., 123-bus system, is conducted to further examine the effectiveness of the proposed algorithm. The configuration of the 123-bus system is shown in Figure 5, and the capacity of the dispatchable DGs and structured microgrids are detailed in Table 5.

With the above interconnected microgrids considered, by solving the optimization problem as shown in the above sections, similar to the results in Table 3, the power generation of each DG is finally converged, as shown in Table 6, and the voltage of the characteristic DG bus is detailed in Table 7. It can be seen that the generated power and voltage magnitude all satisfy the predefined operation constraints, i.e., $0.75 \sim$ 1 p.u. for power generation and $0.99 \sim 1.015$ p.u. for voltage magnitude.

\section{Conclusion}

In this paper, the concept of microgrids has been expanded in a networked connected configuration. The latest operation standards and the functionalities of microgrids are reviewed in detail. Meanwhile, a comprehensive optimization problem is established to derive the optimal operation condition of networked microgrids. A test model is established in MATLAB and numerical study is conducted to determine the optimal operation conditions of networked microgrids. The operation of distribution grids is challenged by microgrids and DGs with increasing penetration level, which will lead to unexpected issues, e.g., voltage violations. In the proposed work, the optimal operation between local microgrids and the central control system in distribution grids is implemented, which is effective in coordinating multiple systems in the control hierarchy in distribution grids. Future work includes the coordination among multiple microgrids in various 
TABLE 5: Dispatchable DGs and microgrids in the 123-bus testing system.

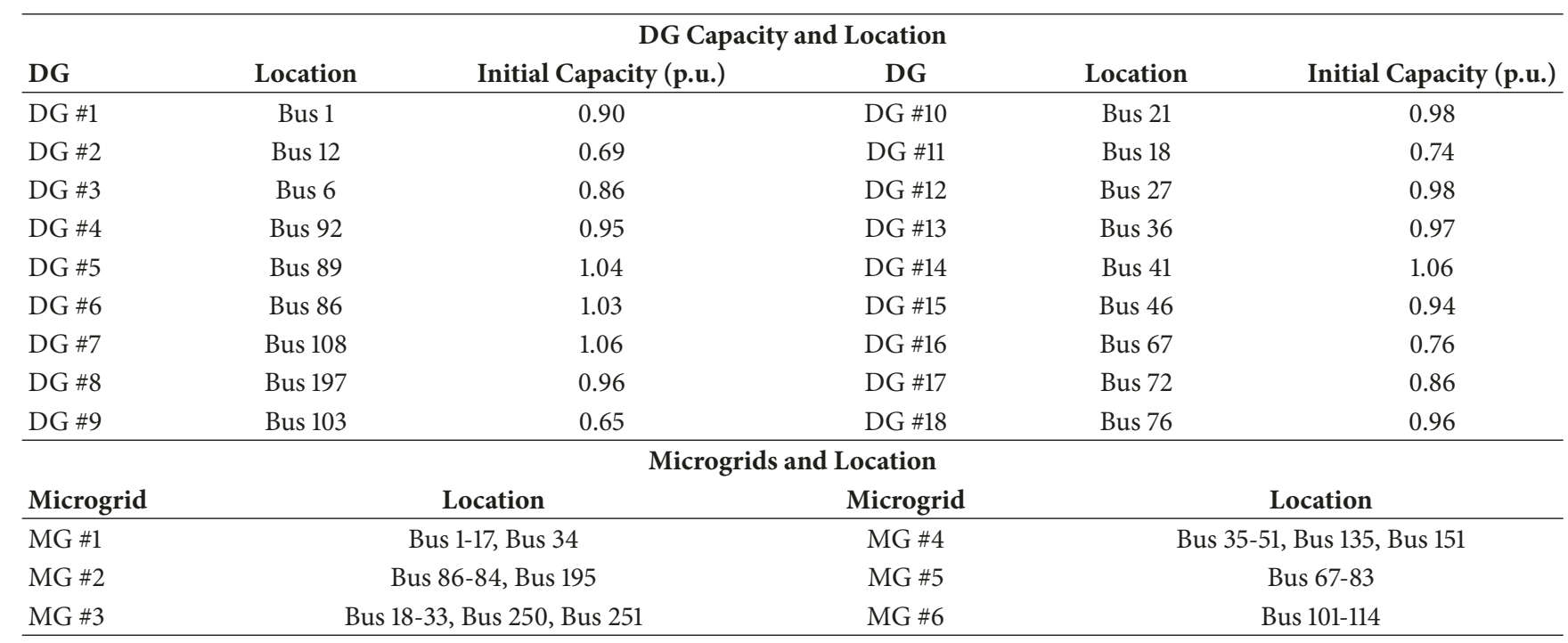

TABLE 6: Power generation of each DG (p.u.), 123-bus system.

\begin{tabular}{lccc}
\hline DG & Output Power (p.u.) & DG & Output Power (p.u.) \\
\hline DG \#1 & 0.92 & DG \#10 & 0.99 \\
DG \#2 & 0.86 & DG \#11 & 0.90 \\
DG \#3 & 0.90 & DG \#12 & 0.98 \\
DG \#4 & 0.96 & DG \#13 & 0.97 \\
DG \#5 & 0.98 & DG \#14 & 0.98 \\
DG \#6 & 0.98 & DG \#15 & 0.96 \\
DG \#7 & 0.99 & DG \#16 & 0.88 \\
DG \#8 & 0.94 & DG \#17 & 0.82 \\
DG \#9 & 0.86 & DG \#18 & 0.90 \\
\hline
\end{tabular}

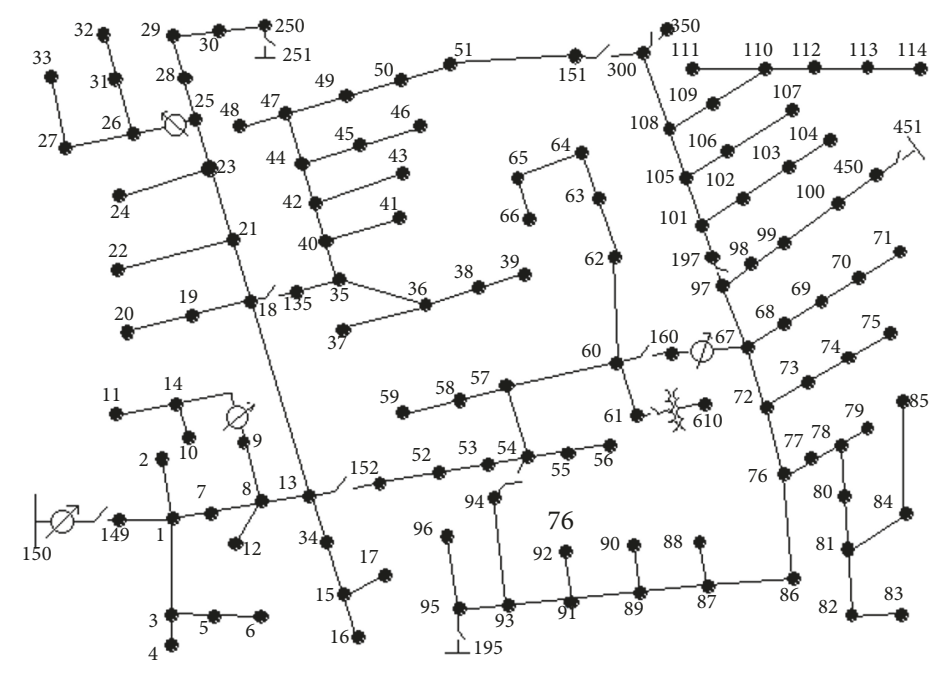

FIGURE 5: 123-bus testing system. 
TABLE 7: Voltage magnitude of each DG (p.u.), 123-bus system.

\begin{tabular}{lccc}
\hline DG & Voltage Mag. (p.u.) & DG & Voltage Mag. (p.u.) \\
\hline DG \#1 & 1.012 & DG \#10 & 1.013 \\
DG \#2 & 0.996 & DG \#11 & 0.992 \\
DG \#3 & 0.998 & DG \#12 & 0.998 \\
DG \#4 & 1.003 & DG \#13 & 0.993 \\
DG \#5 & 1.004 & DG \#14 & 1.012 \\
DG \#6 & 1.004 & DG \#15 & 0.994 \\
DG \#7 & 1.013 & DG \#16 & 0.992 \\
DG \#8 & 1.006 & DG\#17 & 0.992 \\
DG \#9 & 0.994 & DG \#18 & 0.999 \\
\hline
\end{tabular}

grid conditions (balanced and unbalanced fault conditions, etc.) and topologies (i.e., lightly meshed grids). Regulatory constraints should be also considered when further updating the proposed method.

\section{Data Availability}

The data used to support the findings of this study are available from the corresponding author upon request.

\section{Conflicts of Interest}

The authors declare that there are no conflicts of interest regarding the publication of this paper.

\section{References}

[1] D. Baimel, J. Belikov, J. M. Guerrero, and Y. Levron, "Dynamic modeling of networks, microgrids, and renewable sources in the dq0 reference frame: a survey," IEEE Access, vol. 5, pp. 2132321335, 2017.

[2] M. A. Azzouz, H. E. Farag, and E. F. El-Saadany, "Real-time fuzzy voltage regulation for distribution networks incorporating high penetration of renewable sources," IEEE Systems Journal, vol. 11, no. 3, pp. 1702-1711, 2017.

[3] M. S. Hasan, Y. Kouki, T. Ledoux, and J.-L. Pazat, "Exploiting renewable sources: When green SLA becomes a possible reality in cloud computing," IEEE Transactions on Cloud Computing, vol. 5, no. 2, pp. 249-262, 2017.

[4] S. Kazemlou, S. Mehraeen, H. Saberi, and S. Jagannathan, "Stability of the small-scale interconnected DC grids via outputfeedback control," IEEE Journal of Emerging and Selected Topics in Power Electronics, vol. 5, no. 3, pp. 960-970, 2017.

[5] L.-Y. Lu and C.-C. Chu, "Consensus-based secondary frequency and voltage droop control of virtual synchronous generators for isolated ac micro-grids," IEEE Journal on Emerging and Selected Topics in Circuits and Systems, vol. 5, no. 3, pp. 443-455, 2015.

[6] L. Ding, Z. Ma, P. Wall, and V. Terzija, "Graph spectra based controlled islanding for low inertia power systems," IEEE Transactions on Power Delivery, vol. 32, no. 1, pp. 302-309, 2017.

[7] Y. Lu, Q. Wu, Q. Wang, D. Liu, and L. Xiao, "Analysis of a novel zero-voltage-switching bidirectional DC/DC converter for energy storage system," IEEE Transactions on Power Electronics, vol. 33, no. 4, pp. 3169-3179, 2018.
[8] A. Rong and P. B. Luh, "A dynamic regrouping based dynamic programming approach for unit commitment of the transmission-constrained multi-site combined heat and power system," IEEE Transactions on Power Systems, vol. 33, no. 1, pp. 714-722, 2018.

[9] M. Farrokhabadi, B. V. Solanki, C. A. Canizares et al., "Energy storage in microgrids: compensating for generation and demand fluctuations while providing ancillary services," IEEE Power \& Energy Magazine, vol. 15, no. 5, pp. 81-91, 2017.

[10] G. Strbac, N. Hatziargyriou, J. P. Lopes, C. Moreira, A. Dimeas, and D. Papadaskalopoulos, "Microgrids: enhancing the resilience of the european megagrid," IEEE Power \& Energy Magazine, vol. 13, no. 3, pp. 35-43, 2015.

[11] K. Ravindra, B. Kannan, and N. Ramappa, "Microgrids: a valuebased paradigm: the need for the redefinition of microgrids," IEEE Electrification Magazine, vol. 2, no. 1, pp. 20-29, 2014.

[12] Z. Wang, B. Chen, J. Wang, and J. Kim, "Decentralized energy management system for networked microgrids in gridconnected and islanded modes," IEEE Transactions on Smart Grid, vol. 7, no. 2, pp. 1097-1105, 2016. 


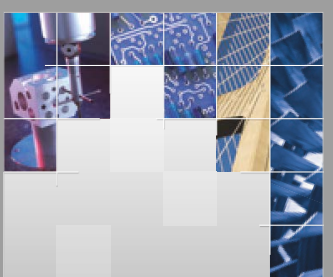

\section{Enfincering}
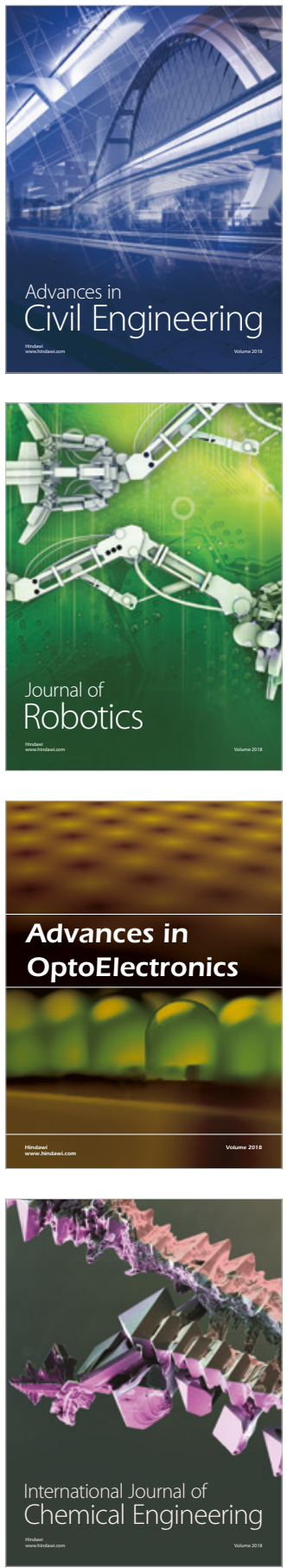

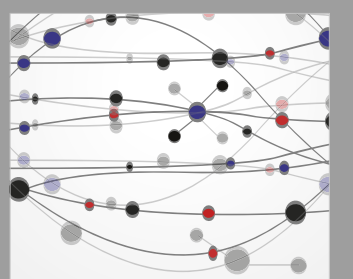

\section{Rotating \\ Machinery}

The Scientific World Journal

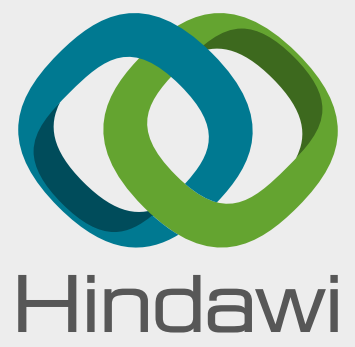

Submit your manuscripts at

www.hindawi.com
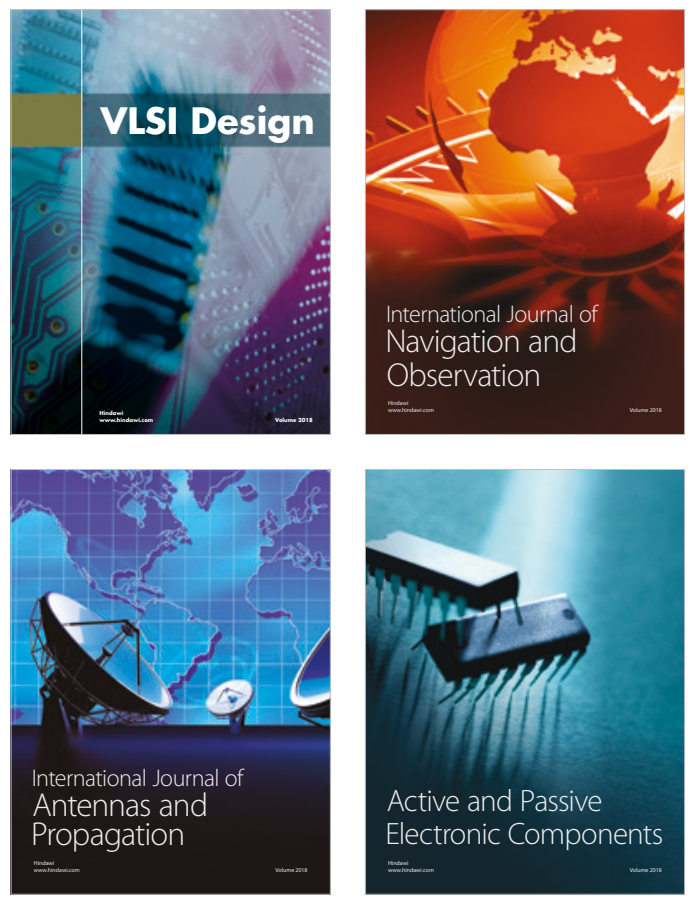
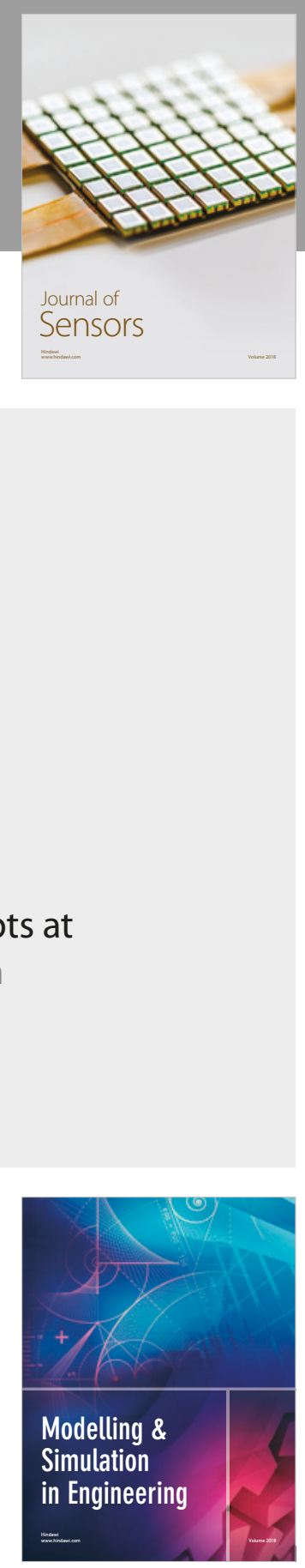

\section{Advances \\ Multimedia}
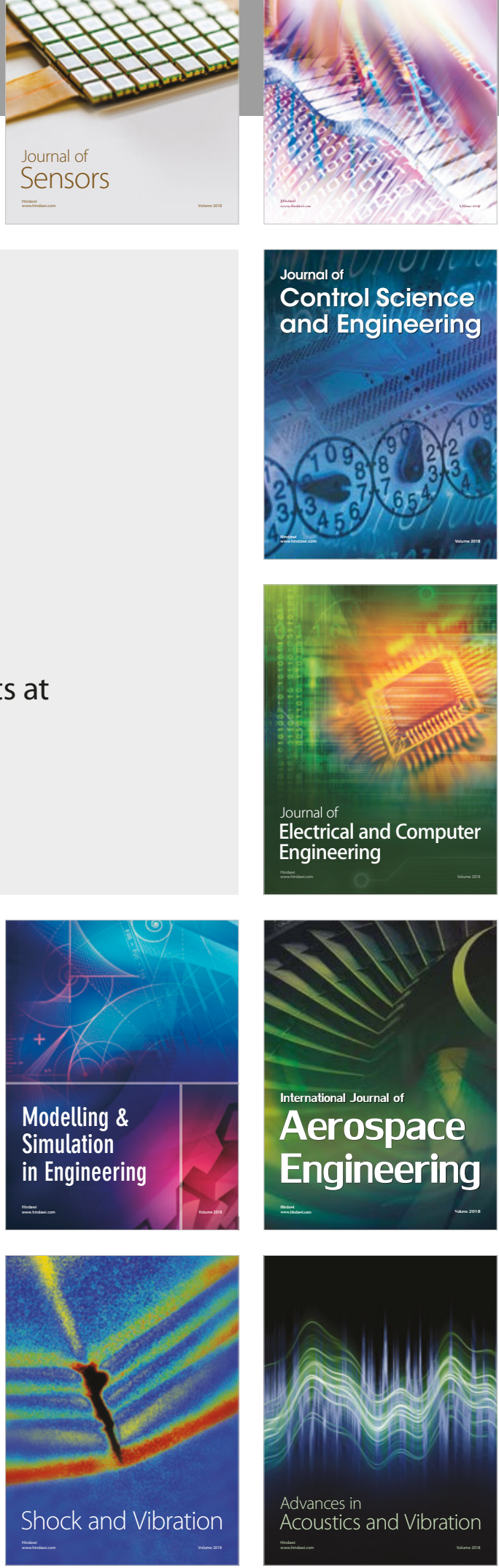